\title{
Expatiating the Therapeutic Profile of Garlic (Allium sativum): a Bench to Bedside Approach
}

\author{
Neelam Sharma ${ }^{1}(\mathbb{D})$, Tapan Behl ${ }^{1}$ (D), Sukhbir Singh ${ }^{1, *}{ }^{\mathbb{D}}$, Anil Bansal ${ }^{1}{ }^{(\mathbb{D})}$, Supriya Kumari Singh ${ }^{1}$ (D), \\ Ishrat Zahoor $^{1}$ (D) \\ 1 Chitkara College of Pharmacy, Chitkara University, Punjab, India \\ * Correspondence: singh.sukhbir12@gmail.com (S.S.);
}

Scopus Author ID: 56402098100

Received: 31.01.2021; Revised: 25.02.2021; Accepted: 2.03.2021; Published: 8.03.2021

\begin{abstract}
Garlic (Allium sativum) is amongst the oldest medicinal plant in centuries. It is an amazing plant that contains numerous bioactive components such as organosulfur compounds, allicin, s-allyl cysteine, S-allyl-mercapto cysteine, diallyl sulfide, diallyl disulfide, and diallyl trisulfide. This review discusses numerous valuable effects and therapeutic potential of garlic in treating several diseases such as cancer, obesity, diabetes, liver injury, hypertension, inflammation, viral infection, and arthritis. The enormous immunomodulatory, anti-oxidant, and anti-microbial characteristics of garlic have also been explained briefly. This review article also gives insight into the nanotechnology-based phytopharmaceuticals of garlic; has summarized pre-clinical models, clinical trials, and patents published about garlic's therapeutic applications and its phytoconstituents in the management of several disease conditions. This review article brings more attention to garlic, providing effective scientific proof for improved utilization of garlic in human health and disease control.
\end{abstract}

Keywords: Allium sativum; allicin; garlic; diallyl sulfide; diallyl disulfide; diallyl trisulfide; organosulfur; s-allyl-mercapto cysteine.

(C) 2021 by the authors. This article is an open-access article distributed under the terms and conditions of the Creative Commons Attribution (CC BY) license (https://creativecommons.org/licenses/by/4.0/).

\section{Introduction}

Garlic (Allium sativum) is the oldest therapeutic plant. It is a wonderful plant that has various bioactive compounds like organosulfur compounds (OSCs), allicin, s-allyl cysteine (SAC), S-allyl-mercapto cysteine (SAMC), diallyl sulfide (DAS), diallyl disulfide (DADS), and diallyl trisulfide (DATS) [1] (Figure 1). Taxonomic details of garlic have been represented in Figure 2. This review discusses various important effects and therapeutic potential of garlic in managing several diseases such as cancer, obesity, diabetes, liver injury, hypertension, inflammation, viral infection, and arthritis. The excellent immunomodulatory, anti-oxidant, and anti-microbial characteristics of garlic have also been described in brief. Traditional phytomedicines of garlic have certain drawbacks like poor aqueous solubility and lesser lipophilicity, causing diminished absorption and bioavailability. However, the application of nanotechnology in the development of nano-based phytopharmaceuticals overcomes these disadvantages. This review article also gives perspective into the nanotechnology-based therapeutics of garlic; has compiled pre-clinical prototypes, clinical trials, and patents reported about the medical strategies of garlic as well as its phytoconstituents in mitigation of many illnesses [2]. 


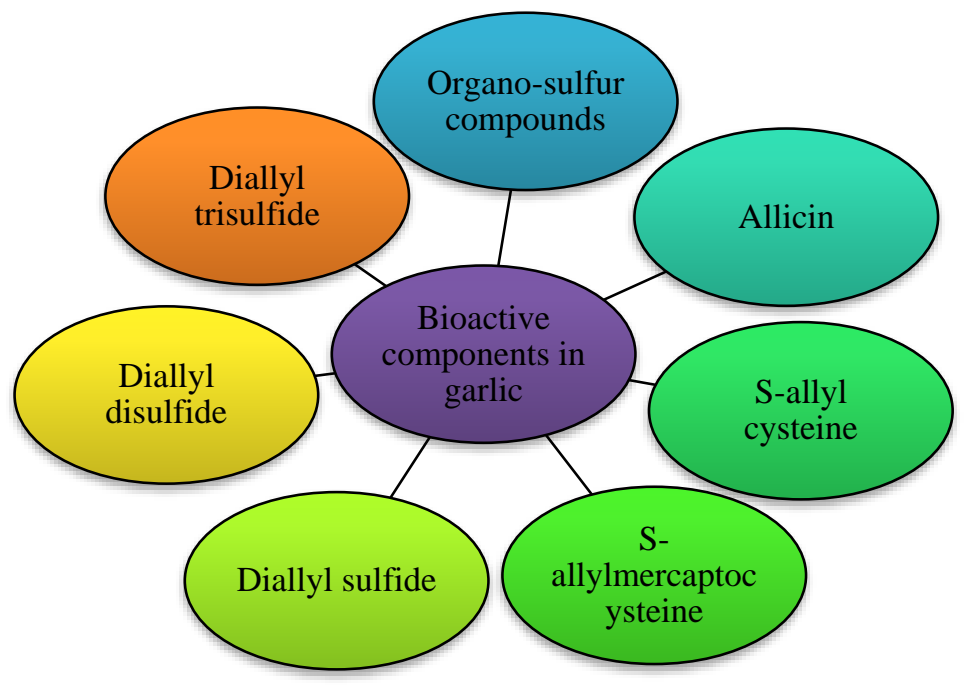

Figure 1. Bioactive compounds in garlic.

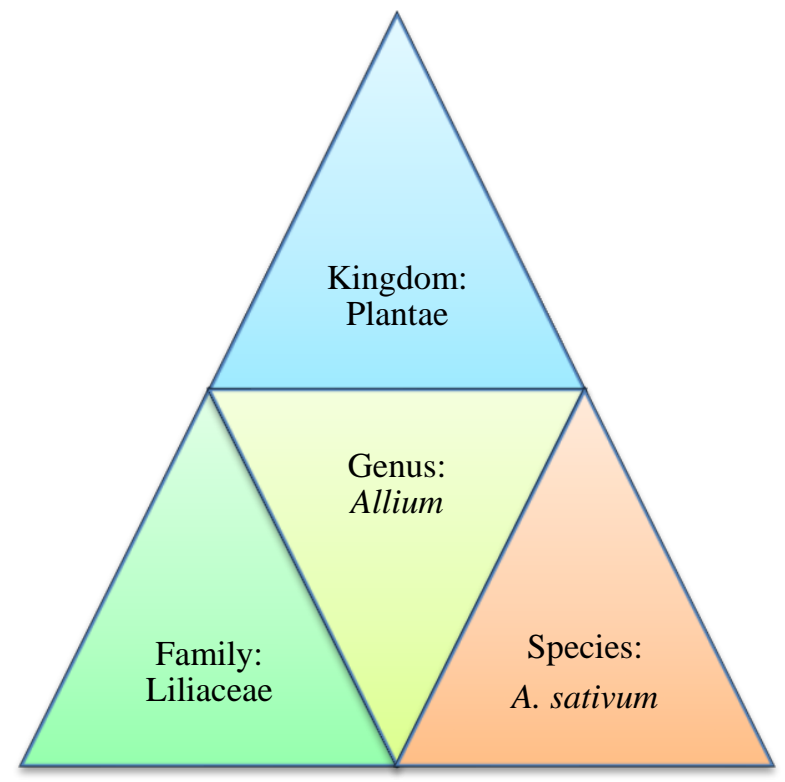

Figure 2. Taxonomic classification of garlic.

\section{Therapeutic Applications of Garlic}

Throughout previous years, garlic has been used to counter several illnesses, as depicted in Figure 3 [2,3].

\subsection{Applications of garlic in cancer therapy.}

Garlic and its constituents are successful in preventing and controlling various cancers $[5,6]$. This research has focused that garlic has been used as a medicinal agent for thousands of years. This has been successfully investigated that SAC and SAMC and some OSCs obtained from aged garlic leaf extract had the highest radical scavenging activity and reported to pervert the expansion of chemically induced as well as transplantable malignancies throughout many laboratory animals that infer that garlic can provide a type of melanoma prevention [7]. A group of researchers documented considerable applications over the use of OSCs isolated from garlic in cancer treatment mitigation as well as chemotherapy [8]. In another study, the activity of allicin in triple-negative breast cancer was discovered [9]. Various chemical compounds found 
in garlic, specifically thioallyl constituents attributed to its anti-carcinogenic properties, and have been shown to impede the covalent binding of toxins to genetic material. They also accelerate cytotoxicity deterioration, have anti-oxidant and radical scavenging characteristics, and control cell proliferation, apoptosis, and immune cells. These have created a new pathway for studies in the area of chemotherapeutic agents and will further investigate the function of garlic in the treatment of health disease [10].

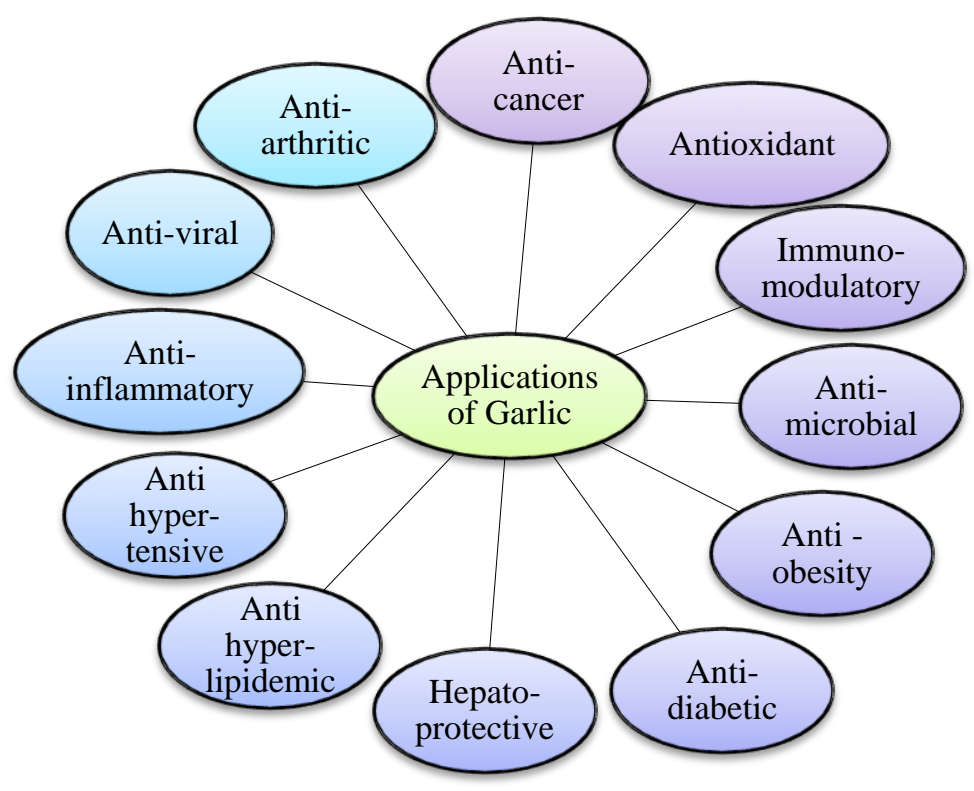

Figure 3. Versatile applications of garlic in several illnesses.

\subsection{Garlic as an excellent anti-oxidant.}

Anti-oxidants play a critical role in establishing balanced free radicals in the body. Superoxide is among the cell's principal free radicals, which changed massively under stressful circumstances [11-13]. Another group of researchers reported that aged garlic extract (AGE) isolated across the longer duration of 20 months is affluent in anti-oxidants, i.e., OSCs, which destroyed free radicals and inhibited oxidative damage [14]. A group of researchers investigated that AGE contains SAC, SAMC, and OSCs with strong anti-oxidative activity [15]. Other groups of researchers investigated the anti-oxidant action of garlic and its phytoconstituents [16-19].

\subsection{Immunomodulatory activity of garlic.}

Garlic displays different bioactive molecules that boost immune function. Polysaccharides and their selenylated form procured from fresh garlic demonstrate a more intense immunomodulation function in contrast to black garlic [20,21]. This was investigated that garlic's IM characteristics are attributed to OSC (allicin), lectins, agglutinins, water-soluble fructans, fructosyl-arginine, SAC, and SAMC [20]. AGE has superior immunomodulatory (IM) properties than fresh garlic, and the effects are being ascribed to the regenerated OSCs $[22,23]$. Alkreathy and his researcher explored that AGE-treated mice survived for a longer time in contrast to control animals. An improved immune reaction in BALB/c mice with fibrosarcoma cancer has been reported with AGE [24]. Another group of researchers examined that AGE considerably lowered antigen-specific ear inflammation. The research implies that AGE might be a suitable choice as an immune modifier in IgE-mediated allergic mouse design [25]. 


\subsection{Anti-microbial potential of garlic.}

Garlic has a broad spectrum of anti-microbial potential, and garlic oil impeded the Penicillium funiculosum fungus, probably invading and damaging the cellular structure $[26,27]$. Besides, raw garlic therapy reduced Helicobacter pylori in the patient's stomach in a clinical trial [28]. Anti-microbial activity of purple and white garlic extracts against oral microbial found that MIC against nine streptococci strains for white and purple garlic was 0.532.0 and $8-64 \mathrm{mg} / \mathrm{ml}$, respectively. It was found that garlic's $2.5 \%$ solution as a mouthwash has bactericidal activity in five-week research in 30 subjects [29]. It has been investigated that ethanolic isolates from fresh garlic have anti-microbial activities against several microorganisms except lactic acid bacteria and yeast [30]. Other groups of researchers investigated the effect of garlic on the oral microbiome [31], explored garlic as an antidote [32], and studied the anti-biofilm effect of nanoparticles loaded with garlic extract [33].

\subsection{Garlic in obesity and hyperlipidemia.}

Obesity is viewed as a serious public health problem [34]. A group of researchers investigated the anti-obesity action of lactic acid fermented garlic extract (LAFGE) in C57BL/6J mice and discovered that it works through adipose tissue hypertrophy inhibition via restraining adipogenesis [35]. Other groups of researchers investigated the anti-obesity effect of garlic's bio-actives [36], garlic oil [37], black garlic's melanoidins [38], potentiating action of diallyl disulfide on the anti-obesity activity of green tea [39], and activity of garlic supplement on pro-inflammatory factors in obesity [40]. It has been investigated the antiobesity effects of garlic oil in the Sprague-Dawley rat model of high-fat diet-induced obesity and discovered that it could be due to enhanced UCP1 expression [41]. Researchers investigated the antihyperlipidemic and anti-obesity efficacy of garlic in obese patients with diabetes mellitus [42] and garlic oil in high-fat diet-induced hyperlipidemic rats [43].

\subsection{Garlic in management of diabetes mellitus.}

Garlic and bioactive components can be protective against diabetes mellitus. Garlic has a longstanding experience as a natural remedy. The anti-oxidant, anti-inflammatory, and antiglycative activities of garlic are accountable for garlic's involvement in reducing the development of diabetes and the occurrence of diabetes-related complications [44-46]. Researchers explored the beneficial effect of garlic extract in diabetes mellitus through DPP-4 inhibition [47]. Another group of researchers explored that garlic oil nanoemulsion could alleviate diabetes mellitus induced nephropathy [48]. In another study, garlic extract's hypoglycaemic characteristics for mitigation of complications in streptozocin/nicotinamideinduced diabetic rats were investigated [49].

\subsection{Hepatoprotective action of garlic.}

A group of researchers found that novel LAFGE based functional-food (D-18-007) has superior choleretic and hepatoprotective action in acute liver injury [50]. Another group of researchers investigated that single-clove garlic has superior hepatoprotective activity compared to multi-clove garlic in carbon tetrachloride (CCl4)-induced liver injury [51]. In another research, the hepatoprotective effect of fermented black garlic extract in CCl4-inducedinduced acute hepatic injury in mice was examined [52]. 


\subsection{Garlic in management of hypertension.}

A group of researchers examined the blood pressure-lowering activity of garlic in a two-kidney-one-clip (2K1C) hypertensive rat model and identified a negative correlation between garlic intake and blood pressure [53]. A group of researchers investigated that raw garlic extract could be used to treat hypertension, as investigated in $2 \mathrm{~K} 1 \mathrm{C}$ rats [54]. Another group of researchers investigated the anti-hypertension efficacy of garlic [55-57].

\subsection{Garlic as an anti-inflammatory.}

Garlic has an anti-inflammatory action, which could be credited to its high OSC content [36,58]. A group of researchers examined the anti-inflammatory effect of garlic extracts. Through an enzyme-linked immunosorbent assay, it was found that cytokine and chemokine levels were significantly diminished in bronchoalveolar fluid [59]. Another research has investigated that aged garlic extract contains three sulfur amino acids like $S$-allyl cysteine, $S$ 1-propenyl cysteine, and $S$-allyl-mercapto cysteine, which exhibited synergistic activity in mitigating inflammation in human gingival epithelial cells [60].

\subsection{Antiviral activity of garlic.}

Another group of researchers studied the antiviral activity of garlic extract against influenza virus in Madin-Darbey Canin Kidney (cell culture and explored inhibitory effect extract on virus penetration and proliferation [61]. The potential antiviral effect of Garlic oil was investigated against Newcastle disease through incubation of LaSota viral strain along with garlic oil for 1 and $24 \mathrm{hrs}$ and detected viral surface proteins and genome [62].

\subsection{Anti-arthritic activity of garlic.}

A group of researchers investigated that diallyl trisulfide extracted from garlic bulb produced potential anti-arthritic activity in a mouse model of collagen-induced arthritis [63]. Another group of researchers evaluated the potential anti-arthritic activity of DADS (20 and $50 \mathrm{mg} / \mathrm{kg}$ ) in complete Freund's adjuvant-induced arthritic rat model [64].

\section{Limitations of traditional phytomedicines of garlic}

Traditional phytomedicines of garlic demonstrate superior in-vitro therapeutic activity and diminished side effects but show lesser in-vivo efficacy due to poor aqueous solubility, lipophilicity, and improper molecular size in poor absorption and lesser bioavailability [65].

\section{Application of nanotechnology-based phytopharmaceuticals of garlic}

Phyto-pharmaceuticals are natural remedies whose potency is due to one or more active ingredients or plant substances. Since prehistoric times, phytopharmaceuticals are often used for various illnesses. The development of a nano-based formulation of phyto-components provides several benefits such as solubility and bioavailability enhancement defend from toxicity, improvement of pharmacological action, upgrading of stability, and safeguard from chemical/physical degradation [65]. Therefore, garlic phytoconstituents have been incorporated into nanotechnology-based phytopharmaceuticals. Table 1 summarizes various types of recent nano-formulations of garlic, excipients involved, and their outcomes. 
Table 1. Applications of nanotechnology in the production of phytopharmaceuticals of garlic.

\begin{tabular}{|c|c|c|c|}
\hline $\begin{array}{l}\text { Phytoconstituents [Nano- } \\
\text { formulation] }\end{array}$ & Excipients & Outcome & Ref. \\
\hline $\begin{array}{l}\text { Diallyl disulfide } \\
\text { [Niosomes] }\end{array}$ & $\begin{array}{l}\text { Cholesterol, Span 20, Span 40, Span 80, } \\
\text { Dicetyl phosphate }\end{array}$ & $\begin{array}{l}\text { Superior treatment of murine } \\
\text { candidiasis }\end{array}$ & [66] \\
\hline $\begin{array}{l}\text { Diallyl disulfide [Solid } \\
\text { lipid nanoparticle] }\end{array}$ & Palmitic acid, Poloxamer 188 & $\begin{array}{l}\text { Enhanced anticancer effect } \\
\text { through induction of apoptosis }\end{array}$ & [67] \\
\hline $\begin{array}{l}\text { Diallyl disulfide [Solid } \\
\text { lipid nanoparticle] }\end{array}$ & $\begin{array}{l}\text { Palmitic acid, Poloxamer 188, Sodium } \\
\text { dodecyl sulfate, 1-ethyl-3-(3- dimethyl- } \\
\text { aminopropyl) carbodiimide hydrochloride }\end{array}$ & $\begin{array}{l}\text { Promising approach for improving } \\
\text { anti-tumor activity and reduced } \\
\text { off-target effects }\end{array}$ & [68] \\
\hline $\begin{array}{l}\text { Diallyl disulfide } \\
\text { [Liposomes] }\end{array}$ & $\begin{array}{l}\text { Egg phosphatidylcholine, Dioleoyl } \\
\text { Phosphatidyl Ethanolamine, and } \\
\text { Cholesteryl Hemisuccinate }\end{array}$ & $\begin{array}{l}\text { Promising strategy against } \\
\text { DMBA-induced skin papilloma }\end{array}$ & [69] \\
\hline $\begin{array}{l}\text { Diallyl disulfide } \\
\text { [Liposomes] }\end{array}$ & Phosphatidylcholine & $\begin{array}{l}\text { Used in murine candidiasis } \\
\text { treatment by decreasing residual } \\
\text { fungal load }\end{array}$ & [70] \\
\hline Diallyl sulfide [Nanorods] & Egg white, Zinc Oxide & $\begin{array}{l}\text { Promising strategy in the treatment } \\
\text { of dermatitis }\end{array}$ & [71] \\
\hline $\begin{array}{l}\text { Diallyl sulfide [Liposomal } \\
\text { gel] }\end{array}$ & $\begin{array}{l}\text { Phosphatidylcholine, Cholesterol, } \\
\text { Carbopol 970, } 974 \text { and } 980\end{array}$ & $\begin{array}{l}\text { Superior herbal antifungal } \\
\text { composition }\end{array}$ & [72] \\
\hline $\begin{array}{l}\text { Allicin [Novel gel } \\
\text { formulation] }\end{array}$ & $\begin{array}{l}\text { Dehydroxanthan gum, Tocopherol, } \\
\text { Sodium Ascorbyl Phosphate, Polysorbate } \\
\text { 20, Sodium hydroxymethylglycinate and } \\
\text { Geranio }\end{array}$ & $\begin{array}{l}\text { Promising strategy against } \\
\text { bacterial infections }\end{array}$ & [73] \\
\hline $\begin{array}{l}\text { Allicin [Polymeric } \\
\text { nanoparticles] }\end{array}$ & Gelatin, Glycyrrhetinic acid & $\begin{array}{l}\text { Promising strategy for liver cancer } \\
\text { therapy }\end{array}$ & [74] \\
\hline Allicin [Liposomal gel] & $\begin{array}{l}\text { Egg phosphatidylcholine, Soya Lecithin, } \\
\text { and Cholesterol }\end{array}$ & $\begin{array}{l}\text { Promising strategy for targeting of } \\
\text { antifungal drugs }\end{array}$ & [75] \\
\hline Allicin [Bilayer tablet] & $\begin{array}{l}\text { Sodium starch glycolate, Polyvinyl } \\
\text { pyrollidone, Xanthan gum, Hydroxypropyl } \\
\text { methylcellulose K-100 }\end{array}$ & $\begin{array}{l}\text { Used for effective treatment of } \\
\text { hypertension }\end{array}$ & [76] \\
\hline $\begin{array}{l}\text { Allicin [Nanocomposite } \\
\text { hydrogel] }\end{array}$ & $\begin{array}{l}\text { Poly-(acrylic acid-co-acrylamide), } \\
\text { Polyvinyl alcohol, Cloisite } 15 \mathrm{~A}\end{array}$ & Superior wound dressing materials & [77] \\
\hline $\begin{array}{l}\text { Diallyl Trisulfide } \\
\text { [Microemulsion] }\end{array}$ & Cremophor EL & $\begin{array}{l}\text { Promising strategy for i.v. delivery } \\
\text { of diallyl trisulfide }\end{array}$ & [78] \\
\hline
\end{tabular}

\section{Pre-clinical testing of garlic and its phytoconstituents for several therapeutic roles}

Different phytoconstituents from garlic, their therapeutic activity, and in-vivo testing models are represented in Table 2.

Table 2. Pre-clinical testing models for investigation of therapeutic roles of phytoconstituents from garlic.

\begin{tabular}{|c|c|c|c|}
\hline Phytoconstituents & Therapeutic role & Models/Activity & Ref. \\
\hline Thiacremenone & $\begin{array}{l}\text { Anti-inflammatory; } \\
\text { anti-arthritic }\end{array}$ & $\begin{array}{l}\text { Tetradecanoyl-phorbol-13-acetate-induced ear edema; } \\
\text { Carrageenan/mycobacterium butyricum-induced } \\
\text { inflammatory/arthritic model }\end{array}$ & [79] \\
\hline Diallyl disulfide & Anti-arthritic & Complete Freund's adjuvant-induced arthritic rats & [64] \\
\hline $\begin{array}{l}\text { Alliin, s-allyl } \\
\text { cysteine }\end{array}$ & Antioxidant & Trolox equivalent antioxidant capacity assay & [11] \\
\hline Diallyl sulfide & $\begin{array}{l}\text { Anti-oxidant/ } \\
\text { Hepatoprotective }\end{array}$ & Hepatic ischemia-reperfusion injury in a rat model & [80] \\
\hline Diallyl disulfide & Anticancer & Human breast cancer cells in culture & [81] \\
\hline $\begin{array}{l}\text { S-allyl- } \\
\text { mercaptocysteine }\end{array}$ & Anticancer & Gentamicin-induced rat kidney injury model & [82] \\
\hline Allicin & Antihypertensive & Tail-cuff method in hypertensive rats & [83] \\
\hline $\begin{array}{l}\text { Propyl-propane } \\
\text { thiosulfonate }\end{array}$ & $\begin{array}{l}\text { Anti-inflammatory in } \\
\text { intestinal colitis }\end{array}$ & $\begin{array}{l}\text { Dinitrobenzene sulfonic acid and dextran sodium sulfate-induced } \\
\text { mice models of colitis }\end{array}$ & [84] \\
\hline
\end{tabular}




\begin{tabular}{l|l|l|l}
\hline Phytoconstituents & Therapeutic role & Models/Activity & Ref. \\
\hline Allicin & $\begin{array}{l}\text { Anti-microbial; Anti- } \\
\text { viral; Anti- } \\
\text { inflammatory }\end{array}$ & Rodent malaria model Plasmodium yoelii 17XL & {$[85]$} \\
\hline Diallyl trisulfide & $\begin{array}{l}\text { Hepatoprotective; } \\
\text { Antioxidant }\end{array}$ & Pentylenetetrazole-induced seizures mice model & {$[86]$} \\
\hline $\begin{array}{l}\text { Tulbaghia violacea } \\
\text { (Wild garlic) }\end{array}$ & Anti-diabetic & Diabetic rat model & {$[87]$}
\end{tabular}

\section{Clinical trials on garlic and its phytoconstituents in treatment of diseases}

Clinical trials which have been previously conducted on phytoconstituents of garlic for the treatment of specific disease conditions have been collected from the official website of clinical trials (clinicaltrials.gov) and are enlisted in Table 3.

Table 3. Clinical trials on application of phytoconstituents of garlic for the treatment of disease.

\begin{tabular}{|c|c|c|c|c|}
\hline Study title & Disease & Sponsor & Phase & Ref \\
\hline \multicolumn{5}{|l|}{ Allicin } \\
\hline $\begin{array}{l}\text { Investigating the gut microbiota } \\
\text { modulation effects of allicin for } \\
\text { cardiovascular disease protection } \\
\text { and establishing microbiota directed } \\
\text { personalized nutrition guidance with } \\
\text { novel humanized gnotobiotic mice } \\
\text { model, microbial culturomics and } \\
\text { metabolomic technique }\end{array}$ & Atherosclerosis & $\begin{array}{l}\text { National Taiwan University } \\
\text { Hospital }\end{array}$ & $\begin{array}{l}\text { Not } \\
\text { available }\end{array}$ & {$[88]$} \\
\hline $\begin{array}{l}\text { Garlic in hyperlipidemia caused by } \\
\text { HAART }\end{array}$ & $\begin{array}{l}\text { HIV infections } \\
\text { hyper- } \\
\text { cholesterolemia } \\
\text { hypertriglyceridemia } \\
\text { hyperglycemia }\end{array}$ & $\begin{array}{l}\text { National Center for } \\
\text { Complementary and } \\
\text { Integrative Health (NCCIH) }\end{array}$ & Phase-2 & [89] \\
\hline $\begin{array}{l}\text { Identifying the anti-blood-clotting } \\
\text { compounds in garlic }\end{array}$ & $\begin{array}{l}\text { Arteriosclerosis } \\
\text { intracranial } \\
\text { arteriosclerosis } \\
\end{array}$ & $\begin{array}{l}\text { National Center for } \\
\text { Complementary and } \\
\text { Integrative Health }(\mathrm{NCCIH}) \\
\end{array}$ & $\begin{array}{l}\text { Not } \\
\text { Applicable }\end{array}$ & {$[90]$} \\
\hline $\begin{array}{l}\text { Pirfenidone plus m-DDO gel in } \\
\text { moderate and severe acne }\end{array}$ & $\begin{array}{l}\text { Acne vulgaris } \\
\text { superficial mixed } \\
\text { comedonal and } \\
\text { inflammatory }\end{array}$ & University of Guadalajara & $\begin{array}{l}\text { Phase-1 } \\
\text { Phase-2 }\end{array}$ & {$[91]$} \\
\hline $\begin{array}{l}\text { Evaluation of post-operative pain } \\
\text { after vital pulpotomy in primary } \\
\text { molars using allium sativum oil } \\
\text { versus MTA }\end{array}$ & Deep caries & Cairo University & $\begin{array}{l}\text { Not } \\
\text { Applicable }\end{array}$ & {$[92]$} \\
\hline $\begin{array}{l}\text { Dietary intervention in follicular } \\
\text { lymphoma (KLYMF) }\end{array}$ & Follicular lymphoma & Oslo University Hospital & Phase-2 & [93] \\
\hline \multicolumn{5}{|l|}{ Diallyl Sulfide } \\
\hline $\begin{array}{l}\text { Effectiveness of garlic oil in the } \\
\text { treatment of arsenical palmer } \\
\text { keratosis }\end{array}$ & $\begin{array}{l}\text { Chronic arsenic } \\
\text { poisoning }\end{array}$ & $\begin{array}{l}\text { Bangabandhu Sheikh Mujib } \\
\text { Medical University, Dhaka, } \\
\text { Bangladesh }\end{array}$ & Phase-2 & {$[94]$} \\
\hline $\begin{array}{l}\text { To evaluate the efficacy of } \\
\text { DDB/garlic oil in patients with } \\
\text { elevated transaminase chronic liver } \\
\text { disease }\end{array}$ & Chronic liver disease & PharmaKing & Phase-4 & {$[95]$} \\
\hline $\begin{array}{l}\text { Hormone estradiol replacement } \\
\text { therapy additional herbals (WH) }\end{array}$ & Menopause & Triệu, Nguyễn Thị, M.D. & Phase-4 & [96] \\
\hline $\begin{array}{l}\text { Study to evaluate efficacy and safety } \\
\text { of PENNEL capsule in the patients } \\
\text { with chronic liver disease }\end{array}$ & $\begin{array}{l}\text { Chronic Liver } \\
\text { Disease }\end{array}$ & PharmaKing & Phase-3 & [97] \\
\hline
\end{tabular}




\begin{tabular}{|c|c|c|c|c|}
\hline Study title & Disease & Sponsor & Phase & Ref. \\
\hline $\begin{array}{l}\text { Effect of DA- } 5515 \text { (Circulan® Soft } \\
\text { Cap.) in patients with chronic } \\
\text { fatigue symptoms due to impaired } \\
\text { blood circulation }\end{array}$ & Fatigue Symptom & Dong-A Pharmaceutical & Phase-4 & [98] \\
\hline $\begin{array}{l}\text { Study to evaluate efficacy, safety } \\
\text { and tolerability of PENNEL capsule } \\
\text { in the patients with chronic liver } \\
\text { disease }\end{array}$ & Chronic liver disease & PharmaKing & Phase-2 & [99] \\
\hline \multicolumn{5}{|l|}{ Diallyl Disulfide } \\
\hline $\begin{array}{l}\text { Efficacy of pirfenidone plus modd } \\
\text { in diabetic foot ulcers }\end{array}$ & Diabetic foot ulcers & University of Guadalajara & $\begin{array}{l}\text { Phase-1 } \\
\text { Phase-2 }\end{array}$ & [100] \\
\hline \multicolumn{5}{|l|}{ S-Allyl Cysteine } \\
\hline $\begin{array}{l}\text { Effect of a black garlic extract on } \\
\text { cholesterol LDL levels } \\
\text { (ESACTIVO) }\end{array}$ & $\begin{array}{l}\text { Cardiovascular } \\
\text { diseases }\end{array}$ & $\begin{array}{l}\text { Technological Centre of } \\
\text { Nutrition and Health, Spain }\end{array}$ & $\begin{array}{l}\text { Not } \\
\text { applicable }\end{array}$ & [101] \\
\hline
\end{tabular}

\section{Patents published about garlic's phytoconstituents in the management of diseases}

Patents published on the applications of phytoconstituents of garlic to treat specific disease conditions have been collected from the WIPO and are enlisted in Table 4.

Table 4. Patents filed on the application of phytoconstituents of garlic for the treatment of disease.

\begin{tabular}{|c|c|c|c|c|c|}
\hline Publication No. & Title & Purpose/Disease & Applicant & $\begin{array}{l}\text { Date of } \\
\text { Publication }\end{array}$ & Ref. \\
\hline CN104187691 & $\begin{array}{l}\text { Healthcare food } \\
\text { capable of removing } \\
\text { lead in the body }\end{array}$ & $\begin{array}{l}\text { Metabolite discharge of } \\
\text { harmless elements from } \\
\text { the body }\end{array}$ & $\begin{array}{l}\text { Qingdao Jinjiahui } \\
\text { Food Co., Ltd. }\end{array}$ & 10.12 .2014 & [102] \\
\hline US20160338971 & $\begin{array}{l}\text { Nano co- } \\
\text { encapsulation for the } \\
\text { prevention and } \\
\text { treatment of various } \\
\text { disorders }\end{array}$ & $\begin{array}{l}\text { Anti-oxidants and } \\
\text { chemo-preventive of } \\
\text { various vascular, } \\
\text { cardiovascular, } \\
\text { oncological disorders, } \\
\text { and infectious diseases }\end{array}$ & $\begin{array}{l}\text { Shaker A. Mousa } \\
\text { Soad K. Jaouni }\end{array}$ & 24.11 .2016 & [103] \\
\hline CN101524459 & $\begin{array}{l}\text { Oil-in-water type garlic } \\
\text { in-garlic oil sub- } \\
\text { microemulsion as well } \\
\text { as a } \\
\text { method for preparing } \\
\text { the same }\end{array}$ & $\begin{array}{l}\text { Adjuvant therapy of } \\
\text { bacteria resistance, } \\
\text { cardiovascular and } \\
\text { cerebrovascular diseases } \\
\text { and tumors }\end{array}$ & $\begin{array}{l}\text { Shenyang } \\
\text { Wanaipulide } \\
\text { Pharm-tech Co., } \\
\text { Ltd. }\end{array}$ & 09.09 .2009 & [104] \\
\hline US20180168952 & $\begin{array}{l}\text { Composition and } \\
\text { application in baby } \\
\text { lotion thereof }\end{array}$ & $\begin{array}{l}\text { Moisturizer for skin and } \\
\text { reduces redness of skin } \\
\text { allergy }\end{array}$ & $\begin{array}{l}\text { Shanghai } \\
\text { Children's } \\
\text { Nutrition Center } \\
\text { Co., Ltd }\end{array}$ & 21.06 .2018 & [105] \\
\hline CN102727727 & $\begin{array}{l}\text { Oil-in-water type } \\
\text { sodium nitroprusside- } \\
\text { garlic oil nanoemulsion } \\
\text { antihypertensive drug }\end{array}$ & $\begin{array}{l}\text { Antihypertensive } \\
\text { (Half-life of sodium } \\
\text { nitroprusside prolonged, } \\
\text { and frequency of } \\
\text { administration reduced) }\end{array}$ & $\begin{array}{l}\text { Northwest A\&F } \\
\text { University }\end{array}$ & 17.10 .2012 & [106] \\
\hline IN201711041514 & $\begin{array}{l}\text { Topical formulation of } \\
\text { capsiate with aged } \\
\text { garlic extract to prevent } \\
\text { cold injuries }\end{array}$ & $\begin{array}{l}\text { Prevention and } \\
\text { treatment of cold } \\
\text { injuries }\end{array}$ & $\begin{array}{l}\text { Lovely } \\
\text { Professional } \\
\text { University }\end{array}$ & 23.11 .2018 & [107] \\
\hline US20060018982 & $\begin{array}{l}\text { Topical application } \\
\text { composition for } \\
\text { preventing and treating } \\
\text { pediculosis, method of } \\
\text { elaboration and uses } \\
\text { thereof }\end{array}$ & $\begin{array}{l}\text { Prevention and therapy } \\
\text { of pediculosis }\end{array}$ & $\begin{array}{l}\text { Roldan Vicente } \\
\text { Teofilo } \\
\text { Barboza Juan Jose }\end{array}$ & 26.01 .2006 & [108] \\
\hline
\end{tabular}




\begin{tabular}{l|l|l|l|l|l} 
US20050013921 & $\begin{array}{l}\text { Novel multi-component } \\
\text { garlic food supplement }\end{array}$ & $\begin{array}{l}\text { Mitigation of joint } \\
\text { inflammation }\end{array}$ & $\begin{array}{l}\text { Andrea Lynn } \\
\text { Peticca }\end{array}$ & 20.01.2005
\end{tabular}

\section{Conclusions}

Garlic is a wonderful medicinal plant with abundant bioactive constituents like organosulfur compounds, allicin, s-allyl cysteine, S-allylmercaptocysteine, and diallyl sulfide, diallyl disulfide, and diallyl trisulfide. Through this review article, it has been found that garlic has versatile therapeutic potential in several illnesses such as cancer, obesity, diabetes, liver injury, hypertension, inflammation, viral infection as well as arthritis, and it has considerable immunomodulatory, anti-oxidant, and anti-microbial characteristics. This review article explains that the application of nanotechnology in the development of phytopharmaceuticals of garlic could provide superior therapeutic potential. Pre-clinical models, clinical trials, and patents briefly summarized in this review article about garlic's therapeutic applications and its phytoconstituents could bring readers more attention. Conclusively, this review gives prospective about garlic and provides effectual scientific proof for improved garlic utilization in human health and disease control.

\section{Funding}

This review received no external funding.

\section{Acknowledgments}

The authors express gratitude to Chitkara College of Pharmacy, Chitkara University, Punjab, India, for motivational support for this review's compilation.

\section{Conflict of Interest}

The authors declare no conflict of interest.

\section{References}

1. Zhang, Y.; Bai, C.; Shi, W.; Alvarez-Manzo, H.; Zhang, Y. Identification of Essential Oils Including Garlic Oil and Black Pepper Oil with High Activity against Babesia duncani. Pathogens 2020, 9, https://doi.org/10.3390/pathogens9060466.

2. Allison, G.L.; Lowe, G.M.; Rahman, K. Aged Garlic Extract and Its Constituents Inhibit Platelet Aggregation through Multiple Mechanisms. The Journal of Nutrition 2006, 136, 782S-788S, https://doi.org/10.1093/jn/136.3.782S.

3. Shang, A.; Cao, S.-Y.; Xu, X.-Y.; Gan, R.-Y.; Tang, G.-Y.; Corke, H.; Mavumengwana, V.; Li, H.-B. Bioactive Compounds and Biological Functions of Garlic (Allium sativum L.). Foods 2019, 8, https://doi.org/10.3390/foods8070246.

4. Ademiluyi, A.O.; Oboh, G.; Owoloye, T.R.; Agbebi, O.J. Modulatory effects of dietary inclusion of garlic (Allium sativum) on gentamycin-induced hepatotoxicity and oxidative stress in rats. Asian Pacific Journal of Tropical Biomedicine 2013, 3, 470-475, https://doi.org/10.1016/S2221-1691(13)60098-2.

5. Chung, L.Y. The Anti-oxidant Properties of Garlic Compounds: Allyl Cysteine, Alliin, Allicin, and Allyl Disulfide. J. Med. Food 2006, 9, 205-213, https://doi.org/10.1089/jmf.2006.9.205.

6. Ide, N.; Lau, B.H.S. Garlic Compounds Minimize Intracellular Oxidative Stress and Inhibit Nuclear Factor$\kappa \mathrm{B}$ Activation. The Journal of Nutrition 2001, 131, 1020S-1026S, https://doi.org/10.1093/jn/131.3.1020S.

7. Fleischauer, A.T.; Arab, L. Garlic and Cancer: A Critical Review of the Epidemiologic Literature. The Journal of Nutrition 2001, 131, 1032S-1040S, https://doi.org/10.1093/jn/131.3.1032S.

8. Ariga, T.; Seki, T. Antithrombotic and anticancer effects of garlic-derived sulfur compounds: A review. BioFactors 2006, 26, 93-103, https://doi.org/10.1002/biof.5520260201. 
9. Rosas-González, V.C.; Téllez-Bañuelos, M.C.; Hernández-Flores, G.; Bravo-Cuellar, A.; Aguilar-Lemarroy, A.; Jave-Suárez, L.F.; Haramati, J.; Solorzano-Ibarra, F.; Ortiz-Lazareno, P.C. Differential effects of alliin and allicin on apoptosis and senescence in luminal $\mathrm{A}$ and triple-negative breast cancer: Caspase, $\Delta \Psi \mathrm{m}$, and pro-apoptotic gene involvement. Fundam. Clin. Pharmacol. 2020, 34, 671-686, https://doi.org/10.1111/fcp.12559.

10. Zhang, Y.; Liu, X.; Ruan, J.; Zhuang, X.; Zhang, X.; Li, Z. Phytochemicals of garlic: Promising candidates for cancer therapy. Biomed. Pharmacother. 2020, $123, \quad$ 109730, https://doi.org/10.1016/j.biopha.2019.109730.

11. Lee, Y.-M.; Gweon, O.-C.; Seo, Y.-J.; Im, J.; Kang, M.-J.; Kim, M.-J.; Kim, J.-I. Anti-oxidant effect of garlic and aged black garlic in animal model of type 2 diabetes mellitus. Nutr. Res. Pract. 2009, 3, 156-161, https://doi.org/10.4162/nrp.2009.3.2.156.

12. Percival, S.S. Aged Garlic Extract Modifies Human Immunity. The Journal of Nutrition 2016, 146, 433S436S, https://doi.org/10.3945/jn.115.210427.

13. Cheng, H.; Huang, G.; Huang, H. The anti-oxidant activities of garlic polysaccharide and its derivatives. Int. J. Biol. Macromol. 2020, 145, 819-826, https://doi.org/10.1016/j.ijbiomac.2019.09.232.

14. Finkel, T.; Holbrook, N.J. Oxidants, oxidative stress and the biology of ageing. Nature 2000, 408, 239-247, https://doi.org/10.1038/35041687.

15. Imai, J.; Ide, N.; Nagae, S.; Moriguchi, T.; Matsuura, H.; Itakura, Y. Antioxidant and radical scavenging effects of aged garlic extract and its constituents. Planta Med. 1994, 60, 417-420, https://doi.org/10.1055/s2006-959522.

16. Atmaca, G. Antioxidant Effects of Sulfur-Containing Amino Acids. Yonsei Med. J. 2004, 45, 776-788, https://doi.org/10.3349/ymj.2004.45.5.776.

17. Panjeshahin, A.; Mollahosseini, M.; Panbehkar-Jouybari, M.; Kaviani, M.; Mirzavandi, F.; Hosseinzadeh, M. Effects of garlic supplementation on liver enzymes: A systematic review and meta-analysis of randomized controlled trials. Phytother. Res. 2020, 34, 1947-1955, https://doi.org/10.1002/ptr.6659.

18. Gao, H.; Huang, G. Preparation and anti-oxidant activity of carboxymethylated garlic polysaccharide. Int. J. Biol. Macromol. 2019, 121, 650-654, https://doi.org/10.1016/j.ijbiomac.2018.10.094.

19. Feng, Y.; Xu, B.; ElGasim A. Yagoub, A.; Ma, H.; Sun, Y.; Xu, X.; Yu, X.; Zhou, C. Role of drying techniques on physical, rehydration, flavor, bioactive compounds and anti-oxidant characteristics of garlic. Food Chem. 2021, 343, 128404, https://doi.org/10.1016/j.foodchem.2020.128404.

20. Arreola, R.; Quintero-Fabián, S.; López-Roa, R.I.; Flores-Gutiérrez, E.O.; Reyes-Grajeda, J.P.; CarreraQuintanar, L.; Ortuño-Sahagún, D. Immunomodulation and Anti-Inflammatory Effects of Garlic Compounds. Journal of Immunology Research 2015, 2015, 401630, https://doi.org/10.1155/2015/401630.

21. Ishikawa, H.; Saeki, T.; Otani, T.; Suzuki, T.; Shimozuma, K.; Nishino, H.; Fukuda, S.; Morimoto, K. Aged Garlic Extract Prevents a Decline of NK Cell Number and Activity in Patients with Advanced Cancer. The Journal of Nutrition 2006, 136, 816S-820S, https://doi.org/10.1093/jn/136.3.816S.

22. Ichikawa, M.; Yoshida, J.; Ide, N.; Sasaoka, T.; Yamaguchi, H.; Ono, K. Tetrahydro- $\beta$-carboline derivatives in aged garlic extract show anti-oxidant properties. J. Nutr. 2006, 136(3), 726S-731S. https://doi.org/10.1093/jn/136.3.726S.

23. Amagase, H. Clarifying the Real Bioactive Constituents of Garlic. The Journal of Nutrition 2006, 136, 716S725S, https://doi.org/10.1093/jn/136.3.716S.

24. Alkreathy, H.; Damanhouri, Z.A.; Ahmed, N.; Slevin, M.; Ali, S.S.; Osman, A.-M.M. Aged garlic extract protects against doxorubicin-induced cardiotoxicity in rats. Food Chem. Toxicol. 2010, 48, 951-956, https://doi.org/10.1016/j.fct.2010.01.005.

25. Abdullah, T.H.; Kirkpatrick, D.V.; Carter, J. Enhancement of natural killer cell activity in AIDS with garlic. J. Oncol. 1989, 21, 52-53.

26. Bradley, J.M.; Organ, C.L.; Lefer, D.J. Garlic-Derived Organic Polysulfides and Myocardial Protection. The Journal of Nutrition 2016, 146, 403S-409S, https://doi.org/10.3945/jn.114.208066.

27. Pranoto, Y.; Salokhe, V.M.; Rakshit, S.K. Physical and antibacte rial properties of alginate-based edible film incorporated with garlic oil. Food Res. Int. 2005, 38, 267-272, https://doi.org/10.1016/j.foodres.2004.04.009.

28. Sivam, G.P. Protection against Helicobacter pylori and Other Bacterial Infections by Garlic. The Journal of Nutrition 2001, 131, 1106S-1108S, https://doi.org/10.1093/jn/131.3.1106S.

29. Rana, S.V.; Pal, R.; Vaiphei, K.; Sharma, S.K.; Ola, R.P. Garlic in health and disease. Nutr. Res. Rev. 2011, 24, 60-71, https://doi.org/10.1017/s0954422410000338. 
30. Byun, P.-H.; Kim, W.-J.; Yoon, S.-K. Effects of extraction conditions on the functional properties of garlic extracts. Korean Journal of Food Science and Technology 2001, 33, 507-513.

31. Hoglund, K.B.; Barnett, B.K.; Watson, S.A.; Melgarejo, M.B.; Kang, Y. Activity of bioactive garlic compounds on the oral microbiome: a literature review. Gen. Dent. 2020, 68, 27-33.

32. Dorrigiv, M.; Zareiyan, A.; Hosseinzadeh, H. Garlic (Allium sativum) as an antidote or a protective agent against natural or chemical toxicities: A comprehensive update review. Phytother. Res. 2020, 34, 1770-1797, https://doi.org/10.1002/ptr.6645.

33. Girish, V.M.; Liang, H.; Aguilan, J.T.; Nosanchuk, J.D.; Friedman, J.M.; Nacharaju, P. Anti-biofilm activity of garlic extract loaded nanoparticles. Nanomed. Nanotechnol. Biol. Med. 2019, 20, 102009, https://doi.org/10.1016/j.nano.2019.04.012.

34. Naukkarinen, J.; Rissanen, A.; Kaprio, J.; Pietiläinen, K.H. Causes and consequences of obesity: the contribution of recent twin studies. Int. J. Obesity 2012, 36, 1017-1024, https://doi.org/10.1038/ijo.2011.192.

35. Lee, H.-S.; Lim, W.-C.; Lee, S.-J.; Lee, S.-H.; Lee, J.-H.; Cho, H.-Y. Antiobesity Effect of Garlic Extract Fermented by Lactobacillus plantarum BL2 in Diet-Induced Obese Mice. J. Med. Food 2016, 19, 823-829, https://doi.org/10.1089/jmf.2016.3674.

36. Quesada, I.; de Paola, M.; Torres-Palazzolo, C.; Camargo, A.; Ferder, L.; Manucha, W.; Castro, C. Effect of Garlic's Active Constituents in Inflammation, Obesity and Cardiovascular Disease. Curr. Hypertens. Rep. 2020, 22, 6, https://doi.org/10.1007/s11906-019-1009-9.

37. Yang, C.; Li, L.; Yang, L.; Lü, H.; Wang, S.; Sun, G. Anti-obesity and Hypolipidemic effects of garlic oil and onion oil in rats fed a high-fat diet. Nutr. Metab. (Lond.) 2018, 15, 43, https://doi.org/10.1186/s12986018-0275-X.

38. Wu, J.; Liu, Y.; Dou, Z.; Wu, T.; Liu, R.; Sui, W.; Jin, Y.; Zhang, M. Black garlic melanoidins prevent obesity, reduce serum LPS levels and modulate the gut microbiota composition in high-fat diet-induced obese C57BL/6J mice. Food Funct. 2020, 11, 9585-9598, https://doi.org/10.1039/D0FO02379E.

39. Bae, J.; Kumazoe, M.; Fujimura, Y.; Tachibana, H. Diallyl disulfide potentiates anti-obesity effect of green tea in high-fat/high-sucrose diet-induced obesity. J. Nutr. Biochem. 2019, 64, 152-161, https://doi.org/10.1016/j.jnutbio.2018.10.014.

40. Dehghani, S.; Alipoor, E.; Salimzadeh, A.; Yaseri, M.; Hosseini, M.; Feinle-Bisset, C.; Hosseinzadeh-Attar, M.J. The effect of a garlic supplement on the pro-inflammatory adipocytokines, resistin and tumor necrosis factor-alpha, and on pain severity, in overweight or obese women with knee osteoarthritis. Phytomedicine 2018, 48, 70-75, https://doi.org/10.1016/j.phymed.2018.04.060.

41. Kagawa, Y.; Ozaki-Masuzawa, Y.; Hosono, T.; Seki, T. Garlic oil suppresses high-fat diet induced obesity in rats through the upregulation of UCP-1 and the enhancement of energy expenditure. Exp. Ther. Med. 2020, 19, 1536-1540, https://doi.org/10.3892/etm.2019.8386.

42. Kumar, R.; Chhatwal, S.; Arora, S.; Sharma, S.; Singh, J.; Singh, N.; Bhandari, V.; Khurana, A. Antihyperglycemic, antihyperlipidemic, anti-inflammatory and adenosine deaminase-lowering effects of garlic in patients with type 2 diabetes mellitus with obesity. Diabetes, metabolic syndrome and obesity: targets and therapy 2013, 6, 49, https://dx.doi.org/10.2147\%2FDMSO.S38888.

43. Faran, S.A.; Asghar, S.; Khalid, S.H.; Khan, I.U.; Asif, M.; Khalid, I.; Farooq Gohar, U.; Hussain, T. Hepatoprotective and renoprotective properties of lovastatin-loaded ginger and garlic oil nanoemulsomes: insights into serum biological parameters. Medicina. 2019, 55(9), 579. https://doi.org/10.3390/medicina55090579.

44. Wild, S.; Roglic, G.; Green, A.; Sicree, R.; King, H. Global Prevalence of Diabetes. Diabetes Care 2004, 27, 1047, https://doi.org/10.2337/diacare.27.5.1047.

45. Wu, H.-Q.; Wei, X.; Yao, J.-Y.; Qi, J.-Y.; Xie, H.-M.; Sang, A.-M.; Jiang, K. Association between retinopathy, nephropathy, and periodontitis in type 2 diabetic patients: a Meta-analysis. International Journal of Ophthalmology 2021, 14, 141, https://dx.doi.org/10.18240\%2Fijo.2021.01.20.

46. Widyahening, I.; Kayode, G.; Wangge, G.; Grobbee, D. Country Characteristics and Variation in Diabetes Prevalence among Asian Countries - an Ecological Study. Journal of the ASEAN Federation of Endocrine Societies 2019, 34, 80-86, https://dx.doi.org/10.15605/jafes.034.01.12.

47. Kalhotra, P.; Chittepu, V.C.S.R.; Osorio-Revilla, G.; Gallardo-Velazquez, T. Phytochemicals in Garlic Extract Inhibit Therapeutic Enzyme DPP-4 and Induce Skeletal Muscle Cell Proliferation: A Possible Mechanism of Action to Benefit the Treatment of Diabetes Mellitus. Biomolecules 2020, 10, https://doi.org/10.3390/biom10020305. 
48. Yuvashree, M.; Ganesh, R.N.; Viswanathan, P. Potential application of nanoemulsified garlic oil blend in mitigating the progression of type 2 diabetes-mediated nephropathy in Wistar rats. 3 Biotech 2020, 10, 272, https://doi.org/10.1007/s13205-020-02262-w.

49. Ziamajidi, N.; Nasiri, A.; Abbasalipourkabir, R.; Sadeghi Moheb, S. Effects of garlic extract on TNF- $\alpha$ expression and oxidative stress status in the kidneys of rats with STZ + nicotinamide-induced diabetes. Pharm. Biol. 2017, 55, 526-531, https://doi.org/10.1080/13880209.2016.1255978.

50. Kim, T.M.; Kim, K.H.; Jo, J.H.; Park, J.; Kwon, Y.S.; Yang, J.H. Hepatoprotective effect of a novel lactic acid-fermented garlic extract functional food product against acute liver injury. Food Science \& Nutrition 2020, 8, 1012-1019, https://doi.org/10.1002/fsn3.1385.

51. Naji, K.M.; Al-Shaibani, E.S.; Alhadi, F.A.; Al-Soudi, S.a.A.; D'souza, M.R. Hepatoprotective and antioxidant effects of single clove garlic against CCl4-induced hepatic damage in rabbits. BMC Complement. Altern. Med. 2017, 17, 411, https://doi.org/10.1186/s12906-017-1916-8.

52. Tsai, J.-C.; Chen, Y.-A.; Wu, J.-T.; Cheng, K.-C.; Lai, P.-S.; Liu, K.-F.; Lin, Y.-K.; Huang, Y.-T.; Hsieh, C.W. Extracts from Fermented Black Garlic Exhibit a Hepatoprotective Effect on Acute Hepatic Injury. Molecules 2019, 24, https://doi.org/10.3390/molecules24061112.

53. Al-Qattan, K.K.; Alnaqeeb, M.A.; Ali, M. The antihypertensive effect of garlic (Allium sativum) in the rat two-kidney-one-clip Goldblatt model. J. Ethnopharmacol. 1999, 66, 217-222, https://doi.org/10.1016/S0378-8741(98)00173-1.

54. Al-Qattan, K.K.; Khan, I.; Alnaqeeb, M.A.; Ali, M. Thromboxane-B2, prostaglandin-E2 and hypertension in the rat 2-kidney 1-clip model: a possible mechanism of the garlic induced hypotension. Prostaglandins, Leukotrienes and Essential Fatty Acids (PLEFA) 2001, 64, 5-10, https://doi.org/10.1054/plef.2000.0232.

55. Sobenin, I.A.; Myasoedova, V.A.; Iltchuk, M.I.; Zhang, D.-W.; Orekhov, A.N. Therapeutic effects of garlic in cardiovascular atherosclerotic disease. Chinese Journal of Natural Medicines 2019, 17, 721-728, https://doi.org/10.1016/S1875-5364(19)30088-3.

56. Martínez-Pizarro, S. Influencia del ajo en la hipertensión arterial. Hipertensión y Riesgo Vascular 2020, 37, 47-49, https://doi.org/10.1016/j.hipert.2019.11.002.

57. Amor, S.; González-Hedström, D.; Martín-Carro, B.; Inarejos-García, A.M.; Almodóvar, P.; Prodanov, M.; García-Villalón, A.L.; Granado, M. Beneficial Effects of an Aged Black Garlic Extract in the Metabolic and Vascular Alterations Induced by a High Fat/Sucrose Diet in Male Rats. Nutrients 2019, 11, https://doi.org/10.3390/nu11010153.

58. Lee, D.Y.; Li, H.; Lim, H.J.; Lee, H.J.; Jeon, R.; Ryu, J.-H. Anti-Inflammatory Activity of Sulfur-Containing Compounds from Garlic. J. Med. Food 2012, 15, 992-999, https://doi.org/10.1089/jmf.2012.2275.

59. Hsieh, C.C.; Peng, W.H.; Tseng, H.H.; Liang, S.Y.; Chen, L.J.; Tsai, J.C. The Protective Role of Garlic on Allergen-Induced Airway Inflammation in Mice. Am. J. Chin. Med. 2019, 47, 1099-1112, https://doi.org/10.1142/S0192415X19500563.

60. Ohtani, M.; Nishimura, T. Sulfur-containing amino acids in aged garlic extract inhibit inflammation in human gingival epithelial cells by suppressing intercellular adhesion molecule-1 expression and IL-6 secretion. Biomed Rep 2020, 12, 99-108, https://doi.org/10.3892/br.2019.1269.

61. Mehrbod, P.; Amini, E.; Tavassoti-Kheiri, M. Antiviral activity of garlic extract on Influenza virus. virusj 2009, 3, 19-23, https://doi.org/10.21859/isv.3.1.19.

62. Hizam, M.M.; Al-Mubarak, F.T.M.; Al-Masoudi, W.A. Antiviral efficacy of garlic oil against newcastle disease virus. Basrah Journal of Veterinary Research 2019, 18.

63. Liang, J.J.; Li, H.R.; Chen, Y.; Zhang, C.; Chen, D.G.; Liang, Z.C.; Shi, Y.Q.; Zhang, L.L.; Xin, L.; Zhao, D.B. Diallyl Trisulfide can induce fibroblast-like synovial apoptosis and has a therapeutic effect on collageninduced arthritis in mice via blocking NF- $\mathrm{B}$ and Wnt pathways. Int. Immunopharmacol. 2019, 71, 132-138, https://doi.org/10.1016/j.intimp.2019.03.024.

64. Chen, Y.; Xue, R.; Jin, X.; Tan, X. Antiarthritic Activity of Diallyl Disulfide against Freund's AdjuvantInduced Arthritic Rat Model. J. Environ. Pathol. Toxicol. Oncol. 2018, 37, https://doi.org/10.1615/JEnvironPatholToxicolOncol.2018027078.

65. Gunasekaran, T.; Haile, T.; Nigusse, T.; Dhanaraju, M.D. Nanotechnology: an effective tool for enhancing bioavailability and bioactivity of phytomedicine. Asian Pacific Journal of Tropical Biomedicine 2014, 4, S1S7, https://doi.org/10.12980/APJTB.4.2014C980.

66. Alam, M.; Zubair, S.; Farazuddin, M.; Ahmad, E.; Khan, A.; Zia, Q.; Malik, A.; Mohammad, O. Development, characterization and efficacy of niosomal diallyl disulfide in treatment of disseminated murine 
$\begin{array}{llllll}\text { candidiasis. Nanomed. Nanotechnol. Biol. Med. 2013, 9, 247-256, } & \text {, }\end{array}$ https://doi.org/10.1016/j.nano.2012.07.004.

67. Talluri, S.V.; Kuppusamy, G.; Karri, V.V.S.R.; Yamjala, K.; Wadhwani, A.; Madhunapantula, S.V.; Pindiprolu, S.S.S. Application of quality-by-design approach to optimize diallyl disulfide-loaded solid lipid nanoparticles. Artificial Cells, Nanomedicine, and Biotechnology 2017, 45, 474-488, https://doi.org/10.3109/21691401.2016.1173046.

68. Siddhartha, V.T.; Pindiprolu, S.K.S.S.; Chintamaneni, P.K.; Tummala, S.; Nandha Kumar, S. RAGE receptor targeted bioconjuguate lipid nanoparticles of diallyl disulfide for improved apoptotic activity in triple negative breast cancer: in vitro studies. Artificial Cells, Nanomedicine, and Biotechnology 2018, 46, 387-397, https://doi.org/10.1080/21691401.2017.1313267.

69. Khan, A.; Shukla, Y.; Kalra, N.; Alam, M.; Ahmad, M.G.; Hakim, S.R.; Owais, M. Potential of Diallyl Sulfide Bearing pH-Sensitive Liposomes in Chemoprevention Against DMBA-Induced Skin Papilloma. Mol. Med. 2007, 13, 443-451, https://doi.org/10.2119/2006-00111.Khan.

70. Rauf, M.A.; Zubair, S.; Ateeq, H.; Dabeer, K.; Pachauri, S.; Ajmal, M.; Owais, M. Synergistic Effect of Diallyl Sulfide With Zinc Oxide Nanorods: A Novel and Effective Approach for Treatment of Acute Dermatitis in Model Animals. Front. Microbiol. 2018, 9, 586, https://doi.org/10.3389/fmicb.2018.00586.

71. Gupta, S.B.; Bhairy, S.R.; Hirlekar, R.S. Formulation development, characterization and assessment of InVitro antifungal efficacy against Candida albicans of diallyl disulphide liposomal gel using 32 factorial design. Journal of Drug Delivery and Therapeutics 2019, 9, 105-117, https://doi.org/10.22270/jddt.v9i2.2522.

72. Maroof, A.; Farazuddin, M.; Owais, M. Potential use of liposomal diallyl sulfide in the treatment of experimental murine candidiasis. Biosci. Rep. 2010, 30, 223-231, https://doi.org/10.1042/BSR20090068.

73. Cutler, R.R.; Odent, M.; Hajj-Ahmad, H.; Maharjan, S.; Bennett, N.J.; Josling, P.D.; Ball, V.; Hatton, P.; Dall'Antonia, M. In vitro activity of an aqueous allicin extract and a novel allicin topical gel formulation against Lancefield group B streptococci. J. Antimicrob. Chemother. 2009, 63, 151-154, https://doi.org/10.1093/jac/dkn457.

74. Ossama, M.; Hathout, R.M.; Attia, D.A.; Mortada, N.D. Enhanced Allicin Cytotoxicity on HEPG-2 Cells Using Glycyrrhetinic Acid Surface-Decorated Gelatin Nanoparticles. ACS Omega 2019, 4, 11293-11300, https://doi.org/10.1021/acsomega.9b01580.

75. Jamindar, D.; Patidar, N.; Jain, S. Formulation and characterization of allicin-amphotericin-b liposomal gel for the treatment of fungal infections. Journal of Drug Delivery and Therapeutics 2017, 7, 69-70.

76. Das, M.K.; Sahu, B.P.; Hazarika, J.N.R. Development of bilayer tablets for immediate and controlled release of allicin. International Journal of Current Pharmaceutical Research 2017, 9, 153, https://doi.org/10.22159/ijcpr.2017v9i4.20982.

77. Olad, A.; Eslamzadeh, M.; Katiraee, F.; Mirmohseni, A. Evaluation of in vitro anti-fungal properties of allicin loaded ion cross-linked poly (AA-co-AAm)/PVA/Cloisite 15A Nanocomposite hydrogel films as wound dressing materials. Journal of Polymer Research 2020, 27, 100, https://doi.org/10.1007/s10965-020-02072$\mathrm{x}$.

78. Li, X.; Yue, Y.; Zhou, Y.; Fan, Y.; Fan, C.; Huang, Y.; Wu, F.; Liu, Y. An oil-free microemulsion for intravenous delivery of diallyl trisulfide: Formulation and evaluation. Int. J. Pharm. 2011, 407, 158-166, https://doi.org/10.1016/j.ijpharm.2011.01.008.

79. Ban, J.O.; Oh, J.H.; Kim, T.M.; Kim, D.J.; Jeong, H.-S.; Han, S.B.; Hong, J.T. Anti-inflammatory and arthritic effects of thiacremonone, a novel sulfurcompound isolated from garlic via inhibition of NF-kB. Arthrit. Res. Ther. 2009, 11, R145, https://doi.org/10.1186/ar2819.

80. Shaik, I.H.; George, J.M.; Thekkumkara, T.J.; Mehvar, R. Protective Effects of Diallyl Sulfide, a Garlic Constituent, on the Warm Hepatic Ischemia-Reperfusion Injury in a Rat Model. Pharm. Res. 2008, 25, 2231, https://doi.org/10.1007/s11095-008-9601-8.

81. Airo, T.; Yen-Chang, L.; Maki, K.; Norihisa, U.; Katsuhiko, Y. Anticancer Effects of Garlic and Garlicderived Compounds for Breast Cancer Control. Anticancer Agents Med. Chem. 2011, 11, 249-253, https://doi.org/10.2174/187152011795347441.

82. Lv, Y.; So, K.-F.; Wong, N.-K.; Xiao, J. Anti-cancer activities of S-allylmercaptocysteine from aged garlic. Chinese Journal of Natural Medicines 2019, 17, 43-49, https://doi.org/10.1016/S1875-5364(19)30008-1.

83. Cui, T.; Liu, W.; Chen, S.; Yu, C.; Li, Y.; Zhang, J.-Y. Antihypertensive effects of allicin on spontaneously hypertensive rats via vasorelaxation and hydrogen sulfide mechanisms. Biomed. Pharmacother. 2020, 128, 110240, https://doi.org/10.1016/j.biopha.2020.110240. 
84. Vezza, T.; Algieri, F.; Garrido-Mesa, J.; Utrilla, M.P.; Rodríguez-Cabezas, M.E.; Baños, A.; Guillamón, E.; García, F.; Rodríguez-Nogales, A.; Gálvez, J. The Immunomodulatory properties of propyl-propane thiosulfonate contribute to its intestinal anti-inflammatory effect in experimental colitis. Mol. Nutr. Food Res. 2019, 63, 1800653, https://doi.org/10.1002/mnfr.201800653.

85. Feng, Y.; Zhu, X.; Wang, Q.; Jiang, Y.; Shang, H.; Cui, L.; Cao, Y. Allicin enhances host proinflammatory immune responses and protects against acute murine malaria infection. Malar. J. 2012, 11, 268, https://doi.org/10.1186/1475-2875-11-268.

86. Shaaban, A.A.; El-Agamy, D.S. Cytoprotective effects of diallyl trisulfide against valproate-induced hepatotoxicity: new anticonvulsant strategy. Naunyn-Schmiedeberg's Arch. Pharmacol. 2017, 390, 919-928, https://doi.org/10.1007/s00210-017-1393-0.

87. Moodley, K.; Joseph, K.; Naidoo, Y.; Islam, S.; Mackraj, I. Antioxidant, antidiabetic and hypolipidemic effects of Tulbaghia violacea Harv. (wild garlic) rhizome methanolic extract in a diabetic rat model. BMC Complement. Altern. Med. 2015, 15, 408, https://doi.org/10.1186/s12906-015-0932-9.

88. Investigating the gut microbiota modulation effects of allicin for cardiovascular disease protection and establishing microbiota directed personalized nutrition guidance with novel humanized gnotobiotic mice model, microbial culturomics and metabolomic technique. Available online: https://clinicaltrials.gov/ct2/show/NCT04545879.

89. Garlic in hyperlipidemia caused by HAART. Available online: https://clinicaltrials.gov/ct2/show/NCT00029250.

90. Identifying the anti-blood-clotting compounds in garlic. Available online: https://clinicaltrials.gov/ct2/show/NCT00200785.

91. Pirfenidone plus M-DDO gel in moderate and severe acne. Available online: https://clinicaltrials.gov/ct2/show/NCT03076320.

92. Evaluation of post-operative pain after vital pulpotomy in primary molars using Allium sativum oil versus MTA. Available online: https://clinicaltrials.gov/ct2/show/NCT03908489.

93. Dietary intervention in follicular lymphoma (KLYMF). Available online: https://clinicaltrials.gov/ct2/show/NCT00455416.

94. Effectiveness of garlic oil in the treatment of arsenical palmer keratosis. Available online: https://clinicaltrials.gov/ct2/show/NCT01748669.

95. To Evaluate the Efficacy of DDB/Garlic Oil in Patients With Elevated Transaminase Chronic Liver Disease. Available online: https://clinicaltrials.gov/ct2/show/NCT02347319.

96. Hormone Estradiol Replacement Therapy Additional Herbals (WH). Available online: https://clinicaltrials.gov/ct2/show/NCT02618148.

97. Study to Evaluate Efficacy and Safety of PENNEL capsule in the patients with chronic liver disease. Available online: https://clinicaltrials.gov/ct2/show/NCT01393678.

98. Effect of DA-5515 (Circulan® Soft Cap.) in patients with chronic fatigue symptoms due to impaired blood circulation. Available online: https://clinicaltrials.gov/ct2/show/NCT03542721.

99. Study to evaluate efficacy, safety and tolerability of PENNEL capsule in the patients with chronic liver disease. Available online: https://clinicaltrials.gov/ct2/show/NCT01393665.

100.Efficacy of pirfenidone plus MODD in diabetic foot ulcers. Available online: https://clinicaltrials.gov/ct2/show/NCT02632877.

101.Effect of a black garlic extract on cholesterol LDL levels (ESACTIVO). Available online: https://clinicaltrials.gov/ct2/show/NCT04010565.

102.Zhiqiang, G. Healthcare food capable of removing lead in body. CN104187691, 2014. https://patentscope.wipo.int/search/en/detail.jsf?docId=CN128686937\&_cid=P20-KLFEK5-15966-1.

103.Mousa, S.A.; Jaouni, S.K. Nano co-encapsulation for the prevention and treatment of various disorders. Google Patents: 2016.

104.Shubin, L.; Xiaoying, W.; Na, G.; Hai, H. Oil-in-water type garlicin-garlic oil sub-microemulsion as well as $\begin{array}{lll}\text { method for preparing } & \text { same. } & \text { CN101524459, }\end{array}$ https://patentscope.wipo.int/search/en/detail.jsf?docId=CN83719393\&_cid=P20-KLFEYD-18292-1.

105.Deng, W. Composition and application in baby lotion thereof. Google Patents: 2018.

106. Ouyang Wuqing, Sun Jianghong, Xiang Gaoqing. Oil-in-water type sodium nitroprusside$\begin{array}{llll}\text { garlic oil nanoemulsion antihypertensive } & \text { drug. } & \text { CN102727727, } & 2012 .\end{array}$ https://patentscope.wipo.int/search/en/detail.jsf?docId=CN85356668\&_cid=P20-KLFF2H-18699-1. 
107.Gulati, M.; Singh S.K.; Bhola, D. Topical formulation of capsiate with aged garlic extract to prevent cold injuries. IN201711041514,

2018. https://patentscope.wipo.int/search/en/detail.jsf?docId=IN234238794\&_cid=P20-KLFF3Q-18816-1.

108. Roldan, V.; Barboza, J. Topical application composition for preventing and treating pediculosis, method of elaboration and uses thereof. Google Patents: 2006.

109.Peticca, A. Novel multi-component garlic food supplement. Google Patents: 2005. 